\title{
Galois Conjugated Tensor Fusion Categories and Nonunitary Conformal Field Theory
}

\author{
Laurens Lootens $\odot$, Robijn Vanhove, Jutho Haegeman, and Frank Verstraete \\ Department of Physics and Astronomy, Ghent University, Krijgslaan 281, S9, B-9000 Ghent, Belgium
}

(Received 29 September 2019; accepted 2 March 2020; published 23 March 2020)

\begin{abstract}
We provide a generalization of the matrix product operator formalism for string-net projected entangled pair states (PEPS) to include nonunitary solutions of the pentagon equation. These states provide the explicit lattice realization of the Galois conjugated counterparts of $(2+1)$-dimensional topological quantum field theories, based on tensor fusion categories. Although the parent Hamiltonians of these renormalization group fixed point states are gapless, these states can still be the topological ground states of a gapped non-Hermitian Hamiltonian. We show by example that the topological sectors of the Yang-Lee theory (the nonunitary counterpart of the Fibonacci fusion category) can be constructed, even in the absence of closure under Hermitian conjugation of the basis elements of the Ocneanu tube algebra. The topological sector construction is demonstrated by applying the concept of strange correlators to the YangLee model, giving rise to a nonunitary version of the classical hard hexagon model in the Yang-Lee universality class and obtaining all generalized twisted boundary conditions on a finite cylinder of the Yang-Lee edge singularity. Finally, we construct the PEPS transfer matrix and show that taking the Hermitian conjugate changes the topological phase for these nonunitary string-net models.
\end{abstract}

DOI: 10.1103/PhysRevLett.124.120601

Introduction.-Tensor fusion categories [1] describe nonlocal "symmetries" in both topological quantum field theories (TQFTs) [2,3] and conformal field theories (CFTs) $[4,5]$. On the one hand, lattice systems with nonchiral topological quantum order can be realized by the TuraevViro state sum constructions [6,7] or by string-net wave functions [8]. In these descriptions, the topological order emerges from the condensations of "strings" (Wilson lines) that do not necessarily fuse according to group rules, but more generally according to an algebra. The topological sectors (emerging anyonic excitations), given by the Drinfeld center of the input fusion category [9-12], are practically found by block diagonalizing the Ocneanu tube algebra [13-15]. On the other hand, classical two-dimensional critical systems exhibiting a second-order phase transition are captured in the scaling limit by $(1+1)$ dimensional (rational) CFTs. This connection between TQFTs and CFTs was first envisioned by Witten [2] and developed by Fuchs et al. [16-18] by means of tensor fusion categories. The relation between topological conformal defects in CFTs and topological sectors in TQFTs was shown in the latter works and explicitly established on the lattice using string-net models [19].

In previous work [20], this connection was further developed using the concept of strange correlators (SCs), originally used as a detection mechanism for short-range entangled symmetry protected topological phases [21]. The SC establishes a map from a $(2+1)$-dimensional quantum state $|\Psi\rangle$ to a corresponding classical two-dimensional partition function by means of taking the overlap of the state with an unentangled state $|\Omega\rangle$. We argued that the SC construction for long-range entangled string-net wave functions in terms of projected entangled pair states (PEPS) was useful because it systematically describes the nonlocal symmetries of the topological properties of emergent CFTs [19] in terms of matrix product operators (MPOs). Moreover, the SC allows for the construction of the conformal blocks out of the topological sectors of the string-net wave function, which is typically hard to do $[19,22,23]$. The conformal spins of the emergent CFT are in one-to-one correspondence with the topological spins of the original TQFT [4,24].

The generalized SC construction is a Euclidean counterpart to the anyonic chain models [25-27], in which topological defects have already been considered in the context of generalized twisted CFT partition functions for both the Fibonacci chain [25] and the nonunitary Yang-Lee Galois conjugated version [28]. However, the identification of the topological sectors in the spectra in the nonunitary case has not been discussed, to our knowledge, in the case of a nontrivial twist in the partition function on a torus [29,30]. This requires the full Ocneanu tube algebra as basis elements for the projection onto all topological superselection sectors. We stress that the $(1+1)$-dimensional quantum Hamiltonian has the same MPO symmetry as the SC transfer matrix, since the quantum Hamiltonian can also be constructed out of a physical projection of string-net PEPS tensors, hereby promoting the virtual degrees of freedom in the $(2+1)$ dimensional model to physical degrees of freedom in the quantum Hamiltonian and preserving the MPO symmetry.

We aim in this Letter to demonstrate that the MPO formalism that was developed in [31-34] can be extended 
to nonunitary solutions of the pentagon equation. The PEPS that are built out of its solutions, the $F$ symbols, form a natural representation of string-net wave functions with nonlocal symmetries described by MPOs. We apply the SC construction to the example of $\mathrm{su}(2)_{3}$ by exactly diagonalizing the obtained transfer matrix of the nonunitary version of the hard-hexagon model. This model falls into the YangLee universality class with central charge $c=-22 / 5$ and the nontrivial MPO symmetry corresponds to the topological conformal defect with conformal weight $h=-1 / 5$ [35]. Finally, the conformally twisted spectra are projected onto the topological sectors, by means of the obtained expressions for the idempotents of the Ocneanu tube algebra [13-15], to illustrate their correspondence with the sesquilinear combination of the conformal characters on a torus $[20,29,30]$. Finally, we argue that the Hermitian conjugated wave function is in a different topological phase and demonstrate this numerically.

The motivation for this Letter is twofold. On the TQFT side, there has been some discussion regarding the use of nonunitary CFTs [36], and we aim to introduce the powerful concepts and tools in tensor networks to this problem. On the other hand, nonunitary CFTs are ubiquitous in many nonequilibrium statistical mechanics models. Additionally, we see this Letter as a stepping stone toward the tensor network description of logarithmic CFTs, which have remained elusive both theoretically and numerically. These CFTs arise in many critical geometrical lattice models, of which percolation is the prototypical example.

String nets and the pentagon equation.-The tensor network representation of string-net models on a hexagonal lattice can be constructed by defining the following PEPS tensors out of which the hexagonal lattice can be built [37]:

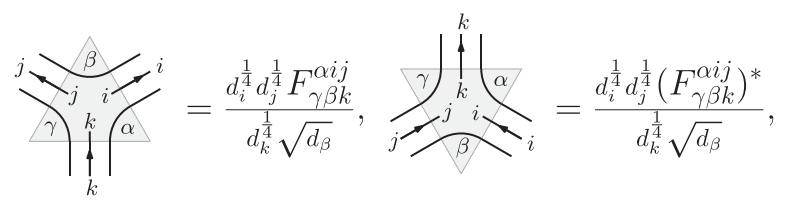

Here, $F_{d e f}^{a b c}$ denotes the $F$ symbol of the input fusion category, also known as the quantum $6 j$ symbol. The nonlocal MPO symmetries of these string-net models are required to satisfy the local pulling-through equation [31-34]

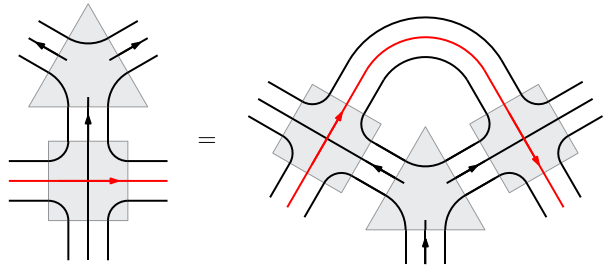

and a similar equation for the second PEPS tensor in Eq. (1). The relative directions of the black and red arrows denote the handedness of the MPO; by convention we call the above MPOs left-handed. If we now define these MPOs as

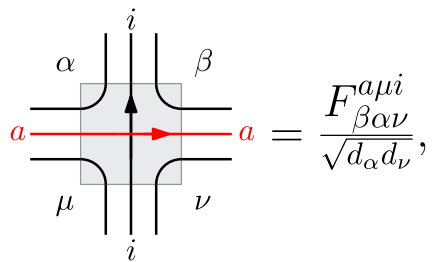

we find that the pulling-through property in Eq. (2) is nothing more than the pentagon equation, which envelops all possible consistency equations and therefore completely determines the $F$ symbols, a property known as Maclane coherence theorem in category theory [24]. Ocneanu rigidity then states that any small deformation of a solution to the pentagon equation can be absorbed through a gauge transformation on the $F$ symbols, so that the solutions fall into discrete families. The MPOs we defined above carry the label $a$, and they form a closed algebra that corresponds to the input fusion category of the string-net model. From a tensor network point of view, the fundamental theorem for matrix product states (which can be trivially extended to MPOs) implies the existence of an object that implements the fusion of two MPOs,

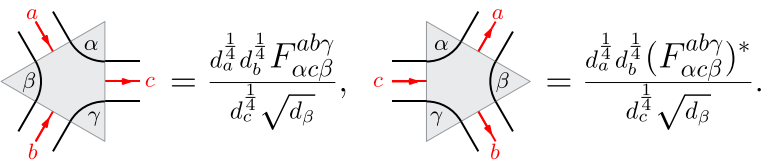

The definitions of the above tensors assume these $F$ symbols to be unitary: $\sum_{f} F_{d e f}^{a b c}\left(F_{d e^{\prime} f}^{a b c}\right)^{*}=\delta_{e e^{\prime}}$, such that, in particular, the fusion of two MPOs is an isometry. In this Letter, we want to lift this unitarity restriction, for it is known that the pentagon equation also admits nonunitary solutions. Solving the pentagon equation is highly nontrivial, but there are algorithms available that are able to find all solutions, be it unitary or not. For certain simple types of fusion categories, however, based on representations of quantum groups, there exist analytic expressions for the $F$ symbols [38], and it is these models that we will consider in the following sections.

Galois conjugates of $\mathrm{su}(2)_{k}$ models.-The fusion categories we consider in this Letter are quantum deformations of $\mathrm{SU}(2)$, where we limit the number of representations to $k+1$ (generalized) angular momenta that take the values $j=0, \frac{1}{2}, 1, \ldots, k / 2$. These fusion categories are denoted as $\mathrm{su}(2)_{k}$. In the language of anyons, taking the tensor product of two representations of $\operatorname{su}(2)_{k}$ corresponds to the fusion of two anyons, where the fusion rules are dictated by the usual $\mathrm{SU}(2)$ tensor product structure, 


$$
j_{1} \times j_{2}=\sum_{j_{3}=\left|j_{1}-j_{2}\right|}^{\min \left(j_{1}+j_{2}, k-j_{1}-j_{2}\right)} j_{3},
$$

with the modification that $j_{3}$ is also bounded by $k-j_{1}-j_{2}$ to ensure associativity of the fusion rules. The explicit solutions to the pentagon equation for all $\operatorname{su}(2)_{k}$ fusion categories have been found using quantum group techniques [39].

The simplest example of a nonunitary fusion category occurs at level $k=3$, where we have four representations $\left\{0, \frac{1}{2}, 1, \frac{3}{2}\right\}$. The fusion rules can be more conveniently expressed as Fib $\otimes \mathbb{Z}_{2}$, where Fib denotes the category with two objects $\{\mathbf{1}, \tau\}$ with $\tau \times \tau=\mathbf{1}+\tau$ as the only nontrivial fusion rule. The $F$ symbols of a fusion category, where the fusion rules factorize into a tensor product, are given by the product of the $F$ symbols of the factors in the tensor product. We will restrict ourselves to the subalgebra of integer representations of su(2) $)_{3}$ with trivial $\mathbb{Z}_{2}$ charge, which have trivial $\mathbb{Z}_{2} F$ symbols. The only nontrivial $F$ symbols are then given by

$$
F_{\tau e f}^{\tau \tau \tau}=\frac{1}{\phi}\left(\begin{array}{cc}
1 & \sqrt{\phi} \\
\sqrt{\phi} & -1
\end{array}\right)
$$

with $\phi=[(1+\sqrt{5}) / 2]$ for the unitary and $\phi^{\prime}=[(1-\sqrt{5}) / 2]$ for the nonunitary case. The process of going from $\phi$ to $\phi^{\prime}$ is known as Galois conjugation, and for this reason, the nonunitary solution is referred to as a Galois conjugate. An important property of these $F$ symbols is that they are invertible in both cases; this means we can replace the Hermitian conjugate of the $F$ symbol in the definition for the unitary PEPS and fusion tensors by the inverse for the nonunitary case.

Ocneanu tube algebra and central idempotents.-In [33], it was shown that the ground-state space of the MPO-injective PEPS is given by the support of the objects $A_{a b c d}$ defined by

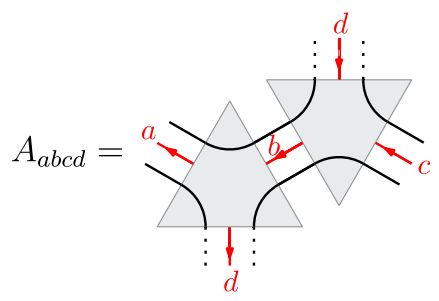

when interpreted as matrices from the indices $c$ to $a$. The dotted lines indicate that they are connected, allowing for an arbitrary numbers of MPO tensors along the connecting line. These objects are basis elements of the Ocneanu tube algebra, and for unitary fusion categories, they were shown to form a $C^{*}$ algebra. This allows the construction of central idempotents that project onto the different topological sectors [40]. When we try to generalize this to nonunitary fusion categories, we find that these objects still form an algebra but are no longer closed under Hermitian conjugation, so that we have no a priori reason to assume the existence of idempotents. Nevertheless, explicit formulas for the idempotents of modular braided categories exist [41] based on their modular $S$ matrices and the $R$ matrices, solutions to the hexagon equations. The nonunitary su $(2)_{k}$ share these properties with their unitary counterparts, and we therefore still expect the existence of idempotents. In this Letter, we take a pragmatic approach and use a constructive algorithm to find the central idempotents, as described in [33]. For the example of the Yang-Lee theory we find four central idempotents labeled $\left\{\mathcal{P}_{1 \overline{1}}, \mathcal{P}_{\tau \overline{1}}, \mathcal{P}_{1 \bar{\tau}}, \mathcal{P}_{\tau \bar{\tau}}\right\}$, three of which are one-dimensional and one is two-dimensional, in agreement with the seven different allowed tubes. These idempotents were shown to correspond to anyonic excitations in the MPO-injective PEPS. The topological spin associated with these anyons [33], which is strongly related to the conformal spin, is given by

$h_{1 \overline{1}}=0, \quad h_{\tau \overline{1}}=-\frac{1}{5}, \quad h_{1 \bar{\tau}}=\frac{1}{5}, \quad h_{\tau \bar{\tau}}=0$.

Strange correlator and Yang-Lee edge singularity.-As mentioned in the Introduction, it was shown in [36] that these nonunitary string-net wave functions cannot have a Hermitian gapped parent Hamiltonian and therefore cannot be topological ground states of a Hermitian Hamiltonian. Our main interest, however, is in the mapping to partition functions of classical statistical mechanics models. We do this by projecting the physical degrees of freedom of the topological PEPS onto a product state, resulting in the partition function of a critical statistical mechanics model described by a CFT. We use exactly the same mapping as in [20] for the unitary Fibonacci model and project all the physical degrees of freedom to $\boldsymbol{\tau}$, resulting in the following Boltzmann weights:

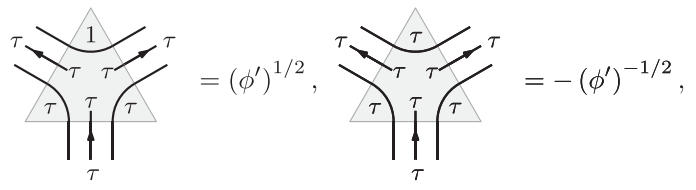

including all rotations and reflections of the PEPS tensors. This model corresponds to the critical hard-hexagon model with negative Boltzmann weights, with the internal $\{1, \tau\}$ loops corresponding to the presence and absence of a particle in the classical model, respectively. It has long been known [42] that, besides a critical fugacity of $z_{c}=\phi^{5}$, this model also is critical at $z_{c}=\left(\phi^{\prime}\right)^{5}$, which is precisely the value we recover from the nonunitary PEPS tensors after applying the strange correlator. The resulting partition function on a cylinder can be diagonalized with or without a flux insertion ( $\tau$ defect), and the spectra can be projected onto the topological sectors using the 

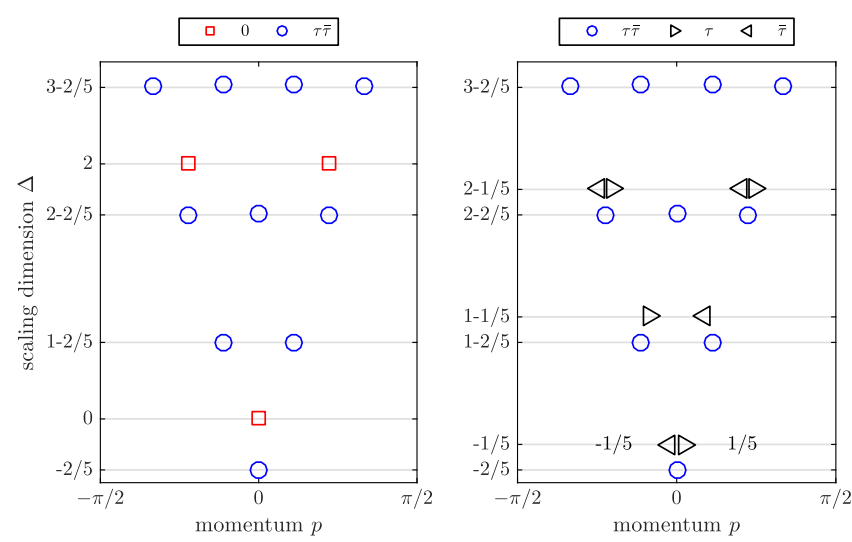

FIG. 1. Topological sector labeling of finite-size CFT spectra (scaling dimension $\Delta$ versus momentum) on a cylinder with a circumference of 18 sites with no defect line on the left and a $\tau$ defect line on the right. The exact topological correction to the conformal spin is denoted next to the first appearance of the respective idempotents.

idempotents discussed above. The results for the trivial flux and $\tau$ flux are shown in Fig. 1, where the positivity of the eigenvalues despite a non-Hermitian transfer matrix allows for the identification with the conformal spectrum of the Yang-Lee edge singularity (YLES). We can make the following correspondence between the topological sectors and the Virasoro characters of the YLES:

$$
\begin{array}{ll}
\mathcal{P}_{1,1 \overline{1}} \rightarrow \chi_{0} \chi_{0}^{*}, & \mathcal{P}_{\tau, \tau \overline{1}} \rightarrow \chi_{-1 / 5} \chi_{0}^{*}, \\
\mathcal{P}_{\tau, 1 \bar{\tau}} \rightarrow \chi_{0} \chi_{-1 / 5}^{*}, & \mathcal{P}_{1, \tau \bar{\tau}}+\mathcal{P}_{\tau, \tau \bar{\tau}} \rightarrow \chi_{-1 / 5} \chi_{-1 / 5}^{*} .
\end{array}
$$

We note that the conformal spectrum without defect has already been obtained in [28] in the context of nonunitary anyonic spin chains, where it was found that the Hamiltonian for these systems possesses a topological symmetry. In the MPO formalism, the local terms of the Hamiltonian of these anyonic spin chains can be written in terms of PEPS tensors as

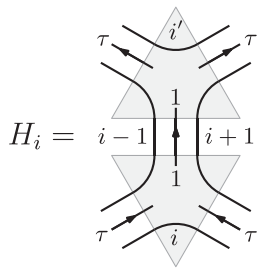

where the topological MPO symmetry is manifest because of the pulling-through equation.

PEPS transfer matrix.-Another object of interest is the PEPS transfer matrix of these nonunitary string-net wave functions. It was shown in [43] that, for the unitary case, these transfer matrices have a degenerate exact fixed point given by the MPO symmetries of the string net. This remains true for the nonunitary case, but one has to be

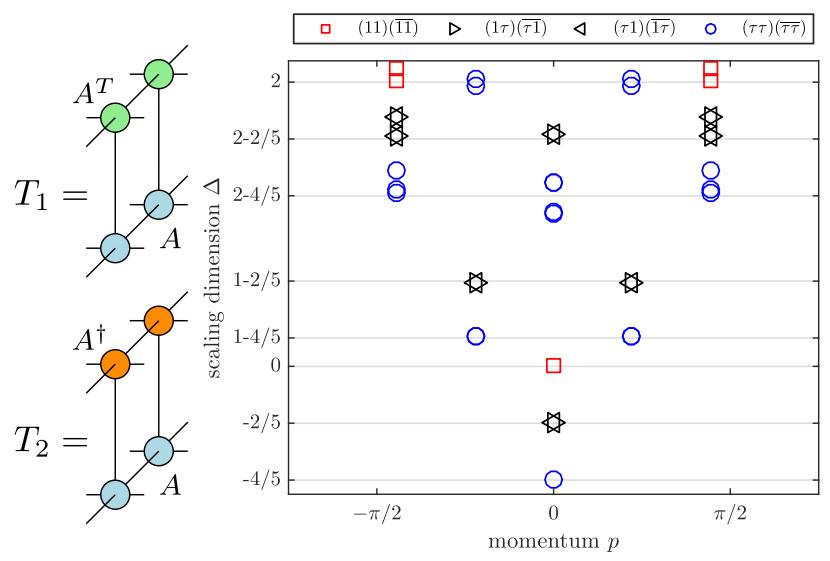

FIG. 2. Definition of the transfer matrices with $A$ as two blocked string-net tensors on the left and YL $\otimes$ YL topological sector labeling of the critical transfer matrix $T_{2}$ on a cylinder of nine sites on the right.

careful with the definition of the upper PEPS layer; the two possibilities are given in Fig. 2. If we use the transpose $\left(T_{1}\right)$, the transfer matrix is gapped with the MPOs as exact fixed points. In contrast, using the usual definition with a Hermitian conjugate $\left(T_{2}\right)$, we verify numerically that this transfer matrix is critical. This is to be expected as the MPO algebra (as a subset of the tube algebra) is not closed under Hermitian conjugation, and therefore the Hermitian conjugated PEPS is in a different topological phase. The transfer can therefore be seen as a generalized strange correlator corresponding to the coset construction in CFT [44]. We could use a fixed point algorithm such as the variational uniform matrix product state algorithm (VUMPS) [45] to obtain the fixed point in the thermodynamic limit and confirm criticality from its entanglement spectrum. However, due to the presence of an excitation with energy lower than the vacuum, VUMPS does not converge since the lowest eigenvalue is not a normalizable state, and we must resort to exact diagonalization in finite size. Doing so, we obtain the YLCFT $\otimes$ YLCFT; the spectrum and topological sector labeling is given in Fig. 2.

Conclusions.-We have generalized the matrix product operator formalism for string-net wave functions to nonunitary solutions of the pentagon equation, provided the underlying category is a braided modular tensor fusion category. We have argued that the construction of the topological superselection sectors (anyons) can still be carried out even though the tube algebra is no longer closed under Hermitian conjugation. This was illustrated numerically by applying the strange correlator map to the YangLee model and obtaining conformal spectra with the explicit identification of the sectors. We also investigated the PEPS transfer matrix properties and found that the conventional definition leads to a critical transfer matrix, of which we identified the CFT and topological sectors. We mentioned the difficulty of using fixed point algorithms for these nonunitary models; we hope to develop new 
numerical methods that are able to deal with these theories in the future. Finally, the obvious extension of this Letter is toward logarithmic CFTs, and we hope to shed new light on old problems, such as percolation. Our approach is built entirely on the existence of nonlocal symmetries in these models and does not a priori require the integrability of these models, the lack of which has been a limiting factor in the deeper understanding of these models.

We would like to thank Dominic Williamson and Nick Bultinck for insightful conversations. This work is supported by an Odysseus grant from the FWO, ERC Grants No. QUTE (647905) and No. ERQUAF (715861), the EU Grant SIQS and FWO PhD Grants (R. V. and L. L.).

[1] P. Etingof, S. Gelaki, D. Nikshych, and V. Ostrik, Tensor Categories (American Mathematical Society, Providence, 2015), Vol. 205.

[2] E. Witten, Topological quantum field theory, Commun. Math. Phys. 117, 353 (1988).

[3] M. Atiyah, Topological quantum field theory, Inst. Hautes Études Sci. Publ. Math. 68, 175 (1988), http://www .numdam.org/item/?id=PMIHES_1988_68_175_0.

[4] G. Moore and N. Seiberg, Classical and quantum conformal field theory, Commun. Math. Phys. 123, 177 (1989).

[5] G. B. Segal, The definition of conformal field theory, in Differential Geometrical Methods in Theoretical Physics, edited by K. Bleuler and M. Werner (Springer Netherlands, Dordrecht, 1988), pp. 165-171.

[6] V. G. Turaev and O. Y. Viro, State sum invariants of 3manifolds and quantum 6j-symbols, Topology 31, 865 (1992).

[7] J. Barrett and B. Westbury, Invariants of piecewise-linear 3manifolds, Trans. Am. Math. Soc. 348, 3997 (1996).

[8] M. A. Levin and X.-G. Wen, String-net condensation: A physical mechanism for topological phases, Phys. Rev. B 71, 045110 (2005).

[9] V. G. Drinfel'd, Quantum groups, J. Sov. Math. 41, 898 (1988).

[10] A. Joyal and R. Street, Tortile Yang-Baxter operators in tensor categories, J. Pure Appl. Algebra 71, 43 (1991).

[11] S. Majid, Representations, duals and quantum doubles of monoidal categories, in Proceedings of the Winter School "Geometry and Physics" (Circolo Matematico di Palermo, 1991), pp. 197-206.

[12] M. Muger, From subfactors to categories and topology II: The quantum double of tensor categories and subfactors, J. Pure Appl. Algebra 180, 159 (2003).

[13] A. Ocneanu, Chirality for operator algebras, Subfactors (Kyuzeso, 1993) (World Scientific, Singapore, 1994), p. 39.

[14] D. Evans and Y. Kawahigashi, On ocneanu's theory of asymptotic inclusions for subfactors, topological quantum field theories and quantum doubles, Int. J. Math. 06, 205 (1995).

[15] D. E. Evans and Y. Kawahigashi, Quantum Symmetries on Operator Algebras (Clarendon Press, Oxford, 1998), Vol. 147.
[16] G. Felder, J. Fröhlich, J. Fuchs, and C. Schweigert, Conformal Boundary Conditions and Three-Dimensional Topological Field Theory, Phys. Rev. Lett. 84, 1659 (2000).

[17] J. Fuchs, I. Runkel, and C. Schweigert, TFT construction of RCFT correlators I: partition functions, Nucl. Phys. B646, 353 (2002).

[18] J. Fröhlich, J. Fuchs, I. Runkel, and C. Schweigert, Kramers-Wannier Duality from Conformal Defects, Phys. Rev. Lett. 93, 070601 (2004).

[19] D. Aasen, R. S. K. Mong, and P. Fendley, Topological defects on the lattice: I. The Ising model, J. Phys. A 49, 354001 (2016).

[20] R. Vanhove, M. Bal, D. J. Williamson, N. Bultinck, J. Haegeman, and F. Verstraete, Mapping Topological to Conformal Field Theories through strange Correlators, Phys. Rev. Lett. 121, 177203 (2018).

[21] Y.-Z. You, Z. Bi, A. Rasmussen, K. Slagle, and C. Xu, Wave Function and Strange Correlator of Short-Range Entangled States, Phys. Rev. Lett. 112, 247202 (2014).

[22] R. S. K. Mong, D. J. Clarke, J. Alicea, N. H. Lindner, and P. Fendley, Parafermionic conformal field theory on the lattice, J. Phys. A 47, 452001 (2014).

[23] M. Hauru, G. Evenbly, W. W. Ho, D. Gaiotto, and G. Vidal, Topological conformal defects with tensor networks, Phys. Rev. B 94, 115125 (2016).

[24] A. Kitaev, Anyons in an exactly solved model and beyond, Ann. Phys. (N.Y.) 321, 2 (2006), January Special Issue.

[25] A. Feiguin, S. Trebst, A. W. W. Ludwig, M. Troyer, A. Kitaev, Z. Wang, and M. H. Freedman, Interacting Anyons in Topological Quantum Liquids: The Golden Chain, Phys. Rev. Lett. 98, 160409 (2007).

[26] C. Gils, E. Ardonne, S. Trebst, A. W. W. Ludwig, M. Troyer, and Z. Wang, Collective States of Interacting Anyons, Edge States, and the Nucleation of Topological Liquids, Phys. Rev. Lett. 103, 070401 (2009).

[27] M. Buican and A. Gromov, Anyonic chains, topological defects, and conformal field theory, Commun. Math. Phys. 356, 1017 (2017).

[28] E. Ardonne, J. Gukelberger, A. W. Ludwig, S. Trebst, and M. Troyer, Microscopic models of interacting Yang-Lee anyons, New J. Phys. 13, 045006 (2011).

[29] V. Petkova and J.-B. Zuber, Generalised twisted partition functions, Phys. Lett. B 504, 157 (2001).

[30] V. B. Petkova and J.-B. Zuber, Conformal boundary conditions and what they teach us, Non-Perturbative QFT Methods and Their Applications (World Scientific, Singapore, 2001), p. 1.

[31] M. B. Şahinoğlu, D. Williamson, N. Bultinck, M. Mariën, J. Haegeman, N. Schuch, and F. Verstraete, Characterizing topological order with matrix product operators, arXiv:1409.2150.

[32] D. J. Williamson, N. Bultinck, M. Mariën, M. B. Şahinoğlu, J. Haegeman, and F. Verstraete, Matrix product operators for symmetry-protected topological phases: Gauging and edge theories, Phys. Rev. B 94, 205150 (2016).

[33] N. Bultinck, M. Mariën, D. J. Williamson, M. B. Şahinoğlu, J. Haegeman, and F. Verstraete, Anyons and matrix product operator algebras, Ann. Phys. (N.Y.) 378, 183 (2017). 
[34] D. J. Williamson, N. Bultinck, and F. Verstraete, Symmetryenriched topological order in tensor networks: Defects, gauging and anyon condensation, arXiv:1711.07982.

[35] P. D. Francesco, P. Mathieu, and D. Senechal, Conformal Field Theory (Springer, New York, 1997) p. 890.

[36] M. H. Freedman, J. Gukelberger, M. B. Hastings, S. Trebst, M. Troyer, and Z. Wang, Galois conjugates of topological phases, Phys. Rev. B 85, 045414 (2012).

[37] O. Buerschaper, M. Aguado, and G. Vidal, Explicit tensor network representation for the ground states of string-net models, Phys. Rev. B 79, 085119 (2009).

[38] E. Ardonne and J. Slingerland, Clebsch-Gordan and 6jcoefficients for rank 2 quantum groups, J. Phys. A 43, 395205 (2010).

[39] A. N. Kirillov and N. Y. Reshetikhin, Representations of the algebra $U_{q}(\mathrm{sl}(2))$, q orthogonal polynomials and invariants of links, Infinite Dimensional Lie Algebras and Groups 7 2851989.

[40] See Supplemental Material at http://link.aps.org/supplemental/ 10.1103/PhysRevLett.124.120601 for the general construc- tion of the Ocneanu tube algebra and the corresponding central idempotents that allow the projection onto the different topological sectors, as well as the explicit forms of these idempotents for the Yang-Lee theory.

[41] R. Koenig, G. Kuperberg, and B. W. Reichardt, Quantum computation with Turaev-Viro codes, Ann. Phys. (N.Y.) 325, 2707 (2010).

[42] R. J. Baxter, Hard hexagons: exact solution, J. Phys. A 13, L61 (1980).

[43] M. Mariën, J. Haegeman, P. Fendley, and F. Verstraete, Condensation-driven phase transitions in perturbed string nets, Phys. Rev. B 96, 155127 (2017).

[44] L. Lootens, R. Vanhove, and F. Verstraete, Cardy states, defect lines and chiral operators of coset CFTs on the lattice, arXiv:1907.02520.

[45] V. Zauner-Stauber, L. Vanderstraeten, M. T. Fishman, F. Verstraete, and J. Haegeman, Variational optimization algorithms for uniform matrix product states, Phys. Rev. B 97, 045145 (2018). 